\title{
Numerical simulation of aerodynamic focusing of particles in supersonic micronozzles
}

\author{
Alexey Kudryavtsev ${ }^{a, b}$ \\ alex@itam.nsc.ru \\ orcid.org/0000-0001-8119-5505 \\ Anton Shershnev ${ }^{a}$ \\ antony@itam.nsc.ru \\ corresponding author \\ orcid.org/0000-0003-0461-2066 \\ Oyuna Rybdylova ${ }^{c}$ \\ o.rybdylova@brighton.ac.uk \\ orcid.org/0000-0001-8541-4197
}

${ }^{a}$ Laboratory of Computational Aerodynamics,

Khristianovich Institute of Theoretical and Applied Mechanics,

Siberian Branch of Russian Academy of Sciences,

4/1 Instituskaya str., 630090 Novosibirsk, Russia

phone number +7 3833308163

${ }^{b}$ Laboratory of Non-equilibrium Flows,

Novosibirsk State University,

2 Pirogova str., 630090, Novosibirsk, Russia

${ }^{c}$ Sir Harry Ricardo Laboratories, Advanced Engineering Centre,

School of Computing, Engineering and Mathematics,

University of Brighton, Brighton BN2 4GJ, UK 


\section{Abstract}

Particle-laden flows in plane, axisymmetric and 3D supersonic micronozzles are investigated numerically using a one-way coupled Eulerian/Lagrangian approach. The carrier gas flow is calculated by solving the Navier-Stokes equations. Rarefaction effects are taken into account by imposing the velocity slip and temperature jump boundary conditions on the nozzle walls. The parameters of the flow around particles are varied in a wide range including hydrodynamic, transitional and free-molecular regimes. It is shown that a collimated beam of particles can be produced using the effect of aerodynamic focusing due to converging flow streamlines in the subsonic part of the nozzle. The collimation is preserved in the supersonic part where the flow is divergent because the rapid drop in the gas density decreases significantly the force acting on the particle. An interesting and unexpected feature of aerodynamic focusing is that the beam collimation is observed in two different ranges of particle sizes. In the first range, for relatively large particles, the collimated beam consists only of particles seeded close to the nozzle axis. In the second range, for smaller particles, the beam includes also a great portion of peripheral particles. The numerical simulation also shows that aerodynamic focusing in a supersonic, convergent-divergent, nozzle enables one to increase significantly the velocity of the collimated beams compared to previously reported results for convergent subsonic nozzles. It may be helpful for technological applications where the aerodynamic scheme of particle focusing can be used (microthrusters, needle-free drug injection, microfabrication, etc.).

Keywords: Gas microflows, Two-phase flow, Aerodynamic focusing

\section{Introduction}

In recent years, the interest in microfluidics has been growing driven by the development of the new micro- and nano-electromechanical systems (MEMS and NEMS) (Ho and Tai, 1998; Gadel-Hak, 1999; Karniadakis et al., 2005). One of the simplest and widely used microdevices is a micronozzle. Flows in micronozzles and exhausting microjets are typically associated with low- 
thrust propulsion systems for orbital maneuvering and trajectory correction of small satellites and spacecraft. However, their potential field of application is vast, from control devices for macroscopic flows to cooling of microchips by a high-speed gas jet.

Because of a very small size of micronozzles (which usually varies from 10 to $1000 \mu \mathrm{m}$ ), the flows in them occur at The boundary layer on the micronozzle walls grows fast and, if the Reynolds number is low enough, it can even lead to deceleration of the flow in the diverging part of the nozzle as was observed in (Rothe, 1971).

Although the first investigations of nozzle flows at low Reynolds numbers were performed relatively long ago (Sutherland and Maes, 1966), the progress in manufacturing of microdevices during the last two decades stimulated new numerical and experimental studies of supersonic micronozzle and microjet flows — see, e. g. Ivanov et al. (1999); Ketsdever et al. (1999); Phalnikar et al. (2008); Aniskin et al. (2011); Sebastião and Santos (2014).

In many potential applications of micronozzles the flow contains not only a carrier gas, but also a dispersed phase in the form of solid particles or liquid droplets. For example, in jet engines (Sternin, 1974) the dispersed phase consists of unburned fuel particles or liquid droplets used for nozzle cooling. A promising practical application of multiphase flows in micronozzles is the cold spray technology (Papyrin et al., 2007), where a gas jet carrying the particulate phase is used to deposit a thin layer of a material on an object placed transversely to the flow. The particle cohesion with a substrate becomes possible due to a plastic deformation of both the particle and the target during the impact. The impact velocity leading to such deformations should be sufficiently high. Typically, the particles are accelerated in the cold spray process during gas expansion in a supersonic convergent-divergent (de Laval) nozzle.

Recently, a new Collimated Aerosol Beam-Direct Write technology (CAB-DW) was proposed for printing solid features on a substrate (Lutfurakhmanov et al., 2010) using precisely collimated particle beams. The lines deposited by CAB-DW are shown to exhibit widths of 
less than $5 \mu \mathrm{m}$. Materials deposition based on the CAB-DW technology has the potential of finding applications in the fields of flexible electronics, sensors, and solar cells.

It is well known that inertial particles in flows with converging streamlines can be focused to a narrow collimated beam owing to aerodynamic focusing (de la Mora and Riesco-Chueca, 1988). The possibility of creating collimated beams of relatively high-speed (up to $100 \mathrm{~m} / \mathrm{s}$ ) particles in a convergent subsonic nozzle was demonstrated both experimentally and numerically by Akhatov et al. (2009) and Lutfurakhmanov et al. (2010). Later, Bhattacharya et al. (2013) investigated numerically the particle-laden flow in a converging-diverging (conical) nozzle and demonstrated, for two different particle diameters, that even higher-speed collimated beams can be obtained using such nozzles. However, the flow conditions considered in their work are restricted by the case when the gas accelerates in the diverging part of the nozzle only for a short distance and the maximum Mach number does not exceed 1.3. After this distance, the gas starts to decelerate so that the jet exhausting from the nozzle is subsonic. The authors explained the flow deceleration by viscous heating and the presence of oblique shock waves. It seems, however, that the main reason was that the wall simulating the substrate was situated very close to the nozzle exit. As a result, it caused a significant pressure increase in the plenum attached to the nozzle exit that influenced also the flow in the diverging part of the nozzle itself.

It is also worth noting that the two-phase flow of a fluid laden with nanoparticles in a supersonic nozzle was also simulated numerically by Kiselev and Kiselev (2009). However, these authors did not consider the phenomenon of aerodynamic focusing.

Thus, the feasibility of aerodynamic focusing and generating collimated particle beams with truly supersonic nozzles, when the jet exhausting the nozzle is supersonic, with the Mach number significantly higher than 1, is still an open question. On one side, the streamlines in the supersonic part of nozzle are divergent, which can defocus the beam. On the other side, 
the gas density in the diverging part is much lower than in the converging part. Therefore, the particles are less affected by the carrier phase, and one can expect that the cluster of particles will retain its structural integrity.

The main goal of the present paper is to investigate this problem numerically in detail and elucidate the feasibility of aerodynamic focusing and generation of a high-speed collimated beam of particles in typical supersonic micronozzle geometries. Some preliminary results concerning beam focusing in plane nozzles were reported earlier by Rybdylova et al. (2013).

As was mentioned in the brief overview of micronozzle studies in the paper by Shershnev and Kudryavtsev (2015), most of the micronozzles are fabricated using photolithography/etching and have rectangular cross sections. Various techniques such as powder blasting with heat treatment and femtosecond laser machining were proposed and tested for fabrication of axisymmetric nozzles (Louwerse, 2009) although, as a rule, precision of shaping is worse and their rotational symmetry is far from ideal. A typical shape of the diverging part is similar to a wedge (in the plane case) or conical (in the axisymmetric case) because of the difficulty of manufacturing contoured nozzles.

Thus, in the paper we consider a 2D flow in a plane wedge-shaped micronozzle and an axisymmetrical flow in a conical mizronozzle. The results of 3D flow computations in micronozzles with a rectangular cross-section are also presented. Numerical simulations are performed for a wide range of particle sizes.

The problems under consideration can be simulated using either of two commonly used approaches. In the two-fluid approach the dispersed phase is treated as another continuum so that Eulerian equations of motion for two interpenetrating media are solved (Nigmatullin, 1990), while in the Lagrangian-Eulerian approach, the dispersed phase is represented by a discrete set of point particles moving according to the forces exerted on them by the carrier phase (Squires, 2007). For our purposes, the Lagrangian-Eulerian approach is quite appropriate because it 
readily allows us to trace particle trajectories and investigate the effects of aerodynamic focusing and collimated beam formation. For this reason, all the computations in the present paper are based on this approach.

In (Bhattacharya et al., 2013) an analysis of comparative importance of different forces acting on a particle in a micronozzle was performed and it was shown that such contributions as the Basset force, the virtual mass force, the pressure gradient force and the buoyancy (gravity) force are negligibly small while the Magnus lifting force is noticeable only for large $(6 \mu \mathrm{m}$ diameter) particles.

The main force acting on a particle immersed in a gas flow is the drag force. In the simplest case it is a well-known Stokes force. In a supersonic nozzle, the flow velocity rapidly grows while its density typically drops by many times. As a result, the flow regimes can change from continuum in the prechamber to rarefied at the nozzle exit. A strong velocity non-equilibrium between the gas and the particles is also possible. The particle Reynolds and Mach number can be rather high and, simultaneously, the Knudsen number can correspond to the transitional and even free-molecular regime. For accurate simulation of particle motion, inertial, compressibility, and rarefaction effects should be taken into account.

A number of approximation formulas for the particle drag coefficient, applicable to a wide range of flow regimes can be found in the literature (Crowe et al., 1973; Henderson, 1976). More recently Stasenko and co-authors published a number of papers on the numerical simulation of two-phase jets, where they proposed and verified expressions for the drag coefficient applicable from creeping to free-molecular flow (Stasenko and Chirikhin, 2002). In the present work the improved expression from Molleson and Stasenko (2008) is used.

Another force that potentially can be of importance for simulation of aerodynamic focusing of particles in the supersonic micronozzle is the Saffman lift force (Saffman, 1965). It only occurs in flows with a transversal gradient of velocity. It is much smaller than the drag force, 
however, acting on the particle transversely to its velocity during the whole travel through the nozzle, the Saffman force can divert it considerably.

Unfortunately, the standard expression for the Saffman force is valid only in the creeping regime. There are no corrections of the Saffman formula taking into account the effects of compressibility and rarefaction in the literature known to the authors while both these effects are of primary importance for the supersonic micronozzle flow. This, in the present study the action of the Saffman force is neglected, but its possible effects deserve further investigation. It is worth noting that in the paper (Bhattacharya et al., 2013) they found that the Saffmann force causes an increase in focusing of the particles.

The rest of the paper is organized as follows: in Sect. 2 we formulate the problem and give a brief description of numerical techniques employed in simulations of the carrier and dispersed phases, in Sect. 3 we present data of numerical simulations of the two-phase flow in micronozzles and discuss results obtained. Some concluding remarks are given in Sect. 4.

\section{Problem statement and numerical techniques}

\subsection{Micronozzle geometry and flow parameters}

The shape of the micronozzle considered in the present study is shown in Fig. 1. It is based on geometrical parameters of the nozzles used in the experiments of Rothe (Rothe, 1971). The nozzle consists of a constant-area section (prechamber), a converging part, and a diverging part. The inclination angles of converging and diverging parts of the nozzle are 45 and 20 degrees, respectively. The nozzle throat half-width is taken equal to $h=100 \mu \mathrm{m}$. The throat is rounded; the radius of rounding $r$ is equal to the throat half-width $h$. The design Mach number at the nozzle exit calculated from $1 \mathrm{D}$ isentropic relations is approximately $\mathrm{M}_{d}=4$ for the plane nozzle and $\mathrm{M}_{d}=7.5$ for the axisymmetric nozzle. A small part of the ambient space near the nozzle 
exit is also included into the computational domain (see Fig. 1).

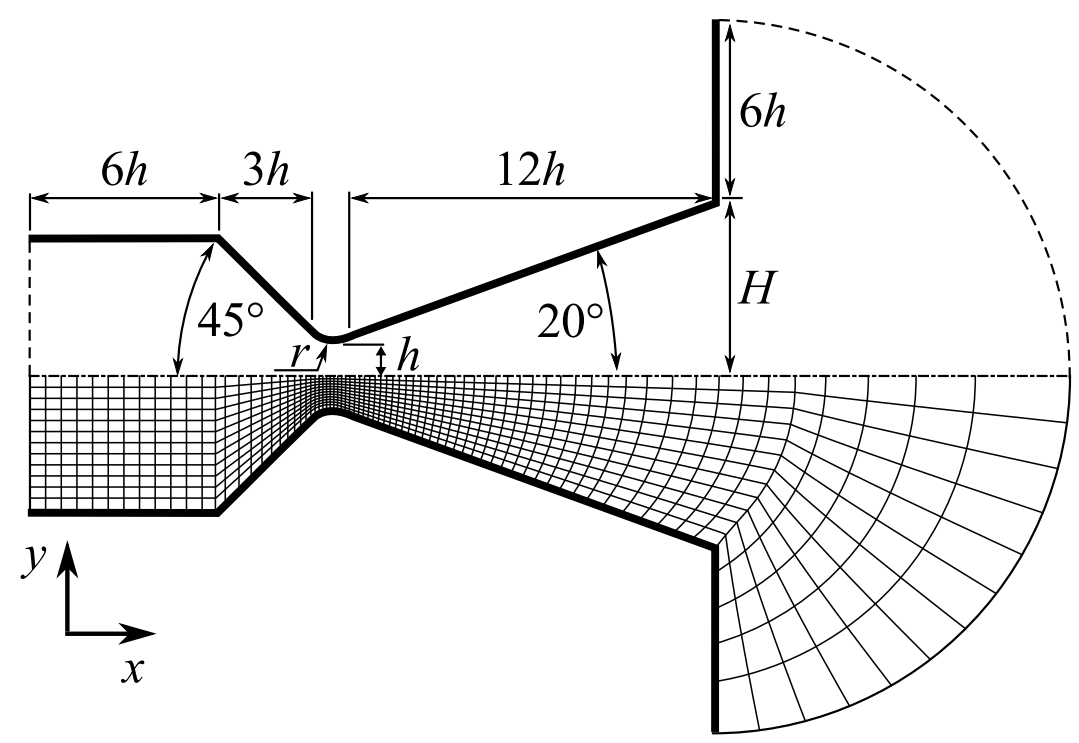

Figure 1: Geometry of the nozzle and computational grid used in carrier gas simulations, every 4th gridline is shown.

An inert monatomic gas (argon) exhausts from the nozzle into vacuum. Both the gas stagnation temperature $T_{0}$ and the nozzle wall temperature $T_{w}$ are taken equal to $300 \mathrm{~K}$. The power-law dependence of gas viscosity on temperature with the exponent equal to 0.81 (Bird, 1994) is assumed. The Reynolds number based on the nozzle throat half-width and density, velocity, and viscosity values at the throat is $R e=350$. In Shershnev and Kudryavtsev (2015), in which both continuum and kinetic simulations were performed, it was shown that the flow in the micronozzle for similar Reynolds number values can be calculated quite accurately using the Navier-Stokes equations with the velocity slip and temperature jump boundary conditions.

It is assumed that the gas carries small spherical solid particles made of a material with the density $\rho_{p}=2000 \mathrm{~kg} / \mathrm{m}^{3}$.

In the present investigation the one-way coupling approach is adopted meaning that the reverse influence of particles on the fluid flow is neglected as well as inter-particle collisions. It is correct for dilute suspensions with low particle mass concentrations. As a consequence, computations can be carried out in two stages. At the first stage, the flow of the carrier 
gas inside the micronozzle is calculated independently of particle movement. At the second stage, the particle trajectories and other particle parameters are determined by integrating their equations of motion using the known carrier gas flowfields for evaluation of forces acting on the particles.

\subsection{Carrier phase simulation}

The governing equations are the Navier-Stokes equations written in a conservative form as

$$
\frac{\partial \mathbf{Q}}{\partial t}+\frac{\partial\left(\mathbf{F}-\mathbf{F}_{\mathbf{v}}\right)}{\partial x}+\frac{\partial\left(\mathbf{G}-\mathbf{G}_{\mathbf{v}}\right)}{\partial y}=\sigma\left(\mathbf{S}_{\mathbf{v}}-\mathbf{S}\right)
$$

Here $\mathbf{Q}$ is the vector of conservative variables, $\mathbf{F}$ and $\mathbf{G}$ are the convective fluxes, $\mathbf{F}_{\mathbf{v}}$ and $\mathbf{G}_{\mathbf{v}}$ are the diffusive fluxes, and $\mathbf{S}$ and $\mathbf{S}_{\mathbf{v}}$ are inviscid and viscous axisymmetric source terms, respectively:

$$
\begin{aligned}
& \mathbf{Q}=\left(\begin{array}{c}
\rho \\
\rho u \\
\rho v \\
E
\end{array}\right), \quad \mathbf{F}=\left(\begin{array}{c}
\rho u \\
\rho u^{2}+p \\
\rho u v \\
(E+p) u
\end{array}\right), \quad \mathbf{G}=\left(\begin{array}{c}
\rho v \\
\rho u v \\
\rho v^{2}+p \\
(E+p) v
\end{array}\right) \\
& \mathbf{F}_{\mathbf{v}}=\left(\begin{array}{c}
0 \\
\tau_{x x} \\
\tau_{x y} \\
\beta_{x}
\end{array}\right), \quad \mathbf{G}_{\mathbf{v}}=\left(\begin{array}{c}
0 \\
\tau_{x y} \\
\tau_{y y} \\
\beta_{y}
\end{array}\right), \quad \mathbf{S}=\left(\begin{array}{c}
\rho v / y \\
\rho u v / y \\
\rho v^{2} / y \\
(E+p) v / y
\end{array}\right), \quad \mathbf{S}_{\mathbf{v}}=\left(\begin{array}{c}
0 \\
\tau_{x y} / y \\
\tilde{\tau} / y \\
\beta_{y} / y
\end{array}\right) \\
& \beta_{x}=u \tau_{x x}+v \tau_{x y}-q_{x}, \quad \beta_{y}=u \tau_{x y}+v \tau_{y y}-q_{y} .
\end{aligned}
$$

The parameter $\sigma=0$ in the $2 \mathrm{D}$ case and $\sigma=1$ in the axisymmetric case.

In Eq. $2 \rho$ is the density, $\mathbf{u} \equiv(u, v)$ is the velocity vector, $E$ is the total energy per unit volume, $p$ is the pressure, $\mathbf{q} \equiv\left(q_{x}, q_{y}\right)=-\kappa \nabla T$ is the heat-flux vector, $T$ is the temperature, and $\kappa$ is the thermal conductivity. The viscous stresses $\tau_{\alpha \beta}$ and $\tilde{\tau}$ are defined as 


$$
\begin{aligned}
\tau_{x x} & =\frac{2}{3} \mu\left(2 \frac{\partial u}{\partial x}-\frac{\partial v}{\partial y}-\sigma \frac{v}{y}\right) \\
\tau_{y y} & =\frac{2}{3} \mu\left(2 \frac{\partial v}{\partial y}-\frac{\partial u}{\partial x}-\sigma \frac{v}{y}\right) \\
\tau_{x y} & =\mu\left(\frac{\partial u}{\partial y}+\frac{\partial v}{\partial x}\right) \\
\tilde{\tau} & =2 \mu\left(\frac{\partial v}{\partial y}-\frac{v}{y}\right)
\end{aligned}
$$

The in-house numerical code CFS3D is employed to solve the Navier-Stokes equations on a structured grid consisting of quadrilateral computational cells. The convective terms are calculated using a TVD (Total Variation Diminishing) shock-capturing scheme based on a MUSCL (Monotonic Upstream-Centered Scheme for Conservation Laws) reconstruction. The values of the flow variables on the cell boundaries are reconstructed from cell-centered values with a $3^{\text {rd }}$ order of accuracy, the minmod slope limiter is applied to avoid spurious oscillations near flow discontinuities. The HLLE (Harten-Lax-van Leer-Einfeldt) approximate Riemann solver is used for evaluating the numerical fluxes from the reconstructed values on two sides of a cell edge. The diffusive terms are calculated with the $2^{\text {nd }}$ order central difference scheme. Time stepping is performed using the explicit Runge-Kutta method of $2^{\text {nd }}$ order.

The CFS3D solver provides an option to the use velocity slip and temperature jump boundary conditions on the solid walls to take into account initial rarefaction effects. The boundary conditions from Kogan (1969)

$$
\left(u_{\tau}\right)_{s}=\alpha_{u} \lambda_{s}\left(\frac{\partial u_{\tau}}{\partial n}\right)_{s}, \quad T_{s}-T_{w}=\alpha_{T} \frac{\gamma}{\gamma-1} \frac{\lambda_{s}}{\operatorname{Pr}}\left(\frac{\partial T}{\partial n}\right)_{s}
$$

were employed in our simulations. Here the subscript $s$ refers to the gas quantities near the wall, $u_{\tau}$ is the velocity component tangential to the wall, $T_{w}$ is the wall temperature, $\lambda$ is the mean free path of gas molecules, $\operatorname{Pr}=2 / 3$ is the Prandtl number, $\gamma=5 / 3$ is the ratio of specific heats, and $n$ is the coordinate normal to the wall. The boundary conditions (4) are deduced from an approximate solution of the kinetic equation in the Knudsen layer. Assuming the diffuse 
reflection of molecules from the wall with complete momentum and energy accommodation, it yields the numerical values of the coefficients $\alpha_{u}=1.142$ and $\alpha_{T}=0.5865$. We consider the scenario when a highly underexpanded jet of the carrier gas exhausts into the region with very low ambient pressure. In this case the size of the jet first shock cell is much larger than the diameter of the nozzle exit cross-section. As a result, the flow is supersonic at the exit boundary of the computational domain. In supersonic outlet all gasdynamic quantities are extrapolated from within the computational domain to the ghost cells. The computations of the carrier phase were performed for the half of domain using the problem symmetry with respect to the $\mathrm{X}$ axis.

\subsection{Dispersed phase simulation}

The dispersed phase simulation is conducted by integrating the equations of motion for each particle:

$$
\begin{aligned}
& \frac{d \mathbf{x}_{p}}{d t}=\mathbf{v}_{p} \\
& m_{p} \frac{d \mathbf{v}_{p}}{d t}=\mathbf{F}_{p}
\end{aligned}
$$

Here $\mathbf{x}_{p}$ and $\mathbf{v}_{p}$ are the coordinate and velocity vectors of the particle, respectively, $m_{p}=\frac{4}{3} \pi \rho_{p} r_{p}^{3}$ is the mass of the particle with the radius $r_{p}$ and density $\rho_{p}$, and $\mathbf{F}_{p}$ is the total force acting on the particle.

The force acting on the particle moving in the fluid in the general case can be divided into several constituents associated with viscous friction, pressure gradient, relative acceleration of the fluid around the particle, and with the unsteady character of the flow (see e.g. Boothroyd (1971)). The latter is becomes significant for the particles subjected to a very strong acceleration, for example, when particle crosses a shock wave. See, e.g., paper by Ling et al. (2011), in which the situation where the unsteady contribution of force can be significant is considered 
in details. However, if the density of the carrier phase is considerably lower than that of the dispersed phase and particles do not interact with shock waves but gradually gain speed due to the accelerating carrier flow, then all of the forces except for drag and, possibly, the Saffman force become negligibly small (see, in particular, an analysis performed in (Bhattacharya et al., 2013)).

As discussed in Introduction, the proper form of the Saffman lift force valid in flow regimes different from the creeping flow is not yet established. It was mentioned in Prosperetti (2007) that in the literature, models in which the Saffman form is adopted way beyond any reasonable domain of validity are not uncommon."

Bhattacharya et al. (2013) used in their computations two different corrections of the Saffman force for higher particle Reynolds numbers suggested by Dandy and Dwyer (Dandy and Dwyer, 1990) and McLaughlin (McLaughlin, 1991), respectively. Unfortunately, these corrections are only applicable if the fluid is incompressible and also they lead to different results: with the former no deviation of particle trajectories was observed while the latter causes a substantial increase in focusing the particles. In the present study, the flow Mach number is significantly higher and compressibility and rarefaction effects much more pronounced. However, there are no known corrections of the Saffman lift force taking into account flow compressibility and rarefaction. With no expression for the lift force valid the flow regimes investigated, we prefer do not include this force in the equations of particle motion.

In fact, it can be hoped that omission of the lift force does not influence on the results concerning beam focusing because the focused particle beam moves near the nozzle axis where transverse flow gradients are small and, consequently, the lift force influence is much smaller than for particles moving closer to the nozzle walls.

The drag force is calculated as

$$
\mathbf{F}_{p}=C_{d} \frac{\pi r_{p}^{2}}{2} \rho\left|\mathbf{v}_{p}-\mathbf{u}\right|\left(\mathbf{v}_{p}-\mathbf{u}\right)
$$


The drag coefficient $C_{d}$ is a function of non-dimensional parameters: the particle Reynolds number $\mathrm{Re}_{p}$, the Mach number $\mathrm{M}_{p}$ of the relative motion of the carrier gas and the particles, and the particle Knudsen number $\mathrm{Kn}_{p}$, which are calculated as

$$
\operatorname{Re}_{p}=\frac{2 r_{p} \rho\left|\mathbf{v}_{p}-\mathbf{u}\right|}{\mu}, \quad \mathrm{M}_{p}=\frac{\left|\mathbf{v}_{p}-\mathbf{u}\right|}{a}, \quad \mathrm{Kn}_{p}=\frac{\lambda}{2 r_{p}}=\sqrt{\frac{\gamma \pi}{2}} \frac{\mathrm{M}_{p}}{\operatorname{Re}_{p}}
$$

Here $a$ is the speed of sound in the location of the particle, and $\lambda=\sqrt{\frac{\pi}{2}} \frac{\mu}{\sqrt{p \rho}}$ is the mean free path.

The specific form of $C_{d}$ is given by the following relation (Molleson and Stasenko, 2008)

$$
C_{d}=A_{c} C_{d}^{c}+A_{r} C_{d}^{r}
$$

where

$$
\begin{aligned}
& C_{d}^{c}=\left(\frac{24}{\mathrm{Re}_{p}}+\frac{4.4}{\sqrt{\mathrm{Re}_{p}}}+0.42\right)\left[1+\exp \left\{-\frac{0.427}{\mathrm{M}_{p}^{4.63}}\right\}\right], \\
& C_{d}^{r}=\frac{\exp \left(-S^{2}\right)}{\sqrt{\pi} S^{3}}\left(1+2 S^{2}\right)+\frac{4 S^{4}+4 S^{2}-1}{2 S^{4}} \operatorname{erf} S+\frac{2}{3} \vartheta \frac{\sqrt{\pi}}{S} \sqrt{\frac{T_{p}}{T}} \\
& A_{c}=(1+K)^{-1}, \quad A_{r}=A_{c} K, \\
& K=\operatorname{Kn}_{p}\left(\left|\mathbf{v}_{p}-\mathbf{u}\right|+\hat{c}\right) \hat{c}^{-1}, \quad \hat{c}=\sqrt{\frac{8 R T}{\pi}}, \quad S=\frac{\left|\mathbf{v}_{p}-\mathbf{u}\right|}{\sqrt{2 R T}} .
\end{aligned}
$$

Here, $c$ and $r$ (both superscripts and subscripts) refer to the drag coefficients for the continuum and free-molecular (rarefied) regimes of flow, respectively, $R$ is the specific gas constant, $\hat{c}$ is the average thermal velocity of molecules, $S$ is the so-called molecular speed ratio equal to $\mathrm{M}_{p} \sqrt{\gamma / 2}$, erf $S$ is the error function, $\vartheta$ is the coefficient of accommodation of momentum of the molecule, $T_{p}$ is the particle surface temperature, and $\mathrm{Kn}_{p}$ is the Knudsen number based on the particle diameter.

Equation (9) bridges two expressions for the drag coefficient in the continuum and freemolecular flows, respectively. The first factor in Eq. (10) for the continuum drag coefficient $C_{d}^{c}$ matches the low-Reynolds number Stokes flow value $C_{d}^{c}=24 / \operatorname{Re}_{p}$ and the high-Reynolds 
number asymptotic value $C_{d}^{c}=0.42$, while the second factor is a correction that allows for compressibility effects. Equation (11) for $C_{d}^{r}$ is the exact analytical expression for the drag coefficient of a sphere in a free-molecular flow. In all computations the accommodation coefficient $\vartheta$ was taken equal to unity and the particle surface temperature $T_{p}$ was assumed to be equal to the gas temperature. A number of computations were repeated with a constant particle temperature condition. It was observed, that in the case considered particle temperature has very small effect on its trajectory and will not change the main conclusions of the study.

Particles are seeded into the flow at the left boundary of the computational domain at different distances from the symmetry line. The starting velocity of a particle is set equal to the gas velocity at the starting point.

To trace the trajectory of a particle fluid properties in the location of the particle are required. To avoid difficulties associated with the particle localization on the grid consisting of arbitrary quadrangles, at the start of the tracing process the fluid flow variables are interpolated onto an auxiliary Cartesian grid. When constructing the Cartesian grid we would like to resolve accurately the flow and particle movements in the throat because it is crucial for further particle tracking so the size of uniform Cartesian cells was taken consistent with that of the finest cells of the boundary-fitted grid. To ensure that the Cartesian grid resolution is sufficient for accurate particle tracking, for some cases the calculations were repeated with a finer Cartesian grid and it was observed that the results were grid-independent.

At each step of integrating Eqs. $(5,6)$ the Cartesian grid cell containing the particle is determined and the flow variables in the particle location are calculated using their values in the grid nodes. Bilinear interpolation is employed for interpolating the flow variables onto the Cartesian grid as well as for finding the fluid properties in the particle location. A second-order Runge-Kutta scheme is utilized to update the particle position and velocity in accordance with the equations of motion $(5,6)$. 
Particles which hit the nozzle walls during the integration can be treated, in general, in different ways. The interaction between the particles and the nozzle wall depends on many factors, such as e. g. the materials of both the nozzle and the particles and the particle velocity. As a result of collision, the particle can stick to the surface loosing both the normal and tangential components of the momentum. Also, it is possible that only the normal component is lost, as a result the particle will slide along the nozzle wall. Specular reflection resulted from an elastic collision is an option too. The most realistic scenario for many cases is a partially inelastic collision when the particle loses a portion of its normal momentum and energy. However, all these possibilities would not affect the aerodynamic focusing of the particles because 1) in the dilute regime collisions of the reflected particles with those in the collimated beam are rare and 2) the normal velocity of the particles colliding with the wall typically is small so that they are reflected at small angles and hardly ever can reach the nozzle axis where collimation is observed. So, in the present paper the simplest possible treatment of the particle-wall interaction is adopted when the particles are removed from the computation after their collision with the wall.

\subsection{Verification of solvers}

To ensure that the solution is grid independent, a series of computation for three different mesh sizes, containing $608 \times 48,1179 \times 96$ and $2184 \times 180$ cells, respectively, was performed. Figure 2 shows the distribution of the non-dimensional gasdynamic parameters along the nozzle centerline. Here and further on, the asterisk $(*)$ subscript denotes the parameters in the nozzle throat calculated from the stagnation parameters using isentropic relations. It is evident that nozzle flow core is predicted correctly even at the lowest spatial resolution. Figure 3 demonstrates the profiles of gasdynamic quantities in the nozzle exit cross-section between the nozzle wall and the core flow and the convergence of the carrier phase solution. 


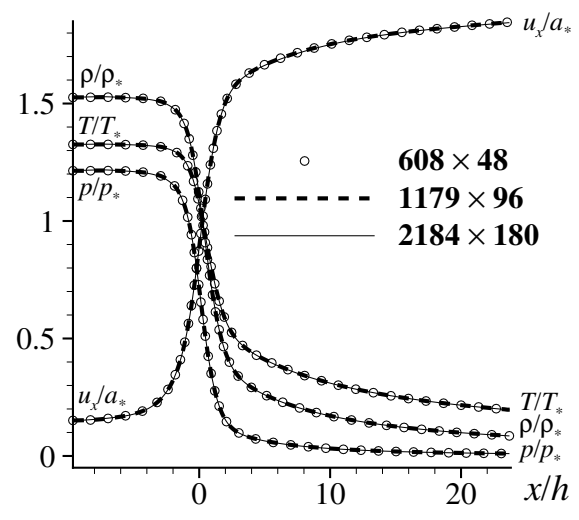

Figure 2: Plane nozzle. Distribution of gasdynamic parameters along the centerline on different meshes.

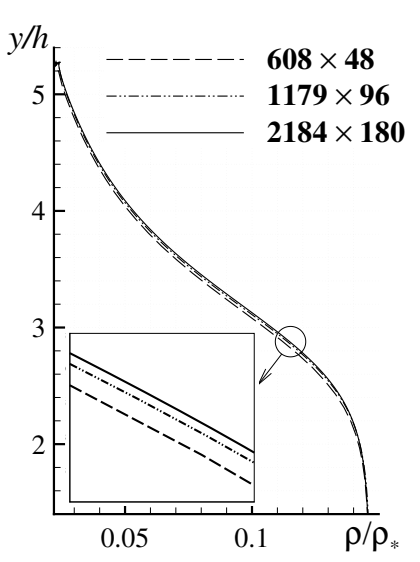

(a)

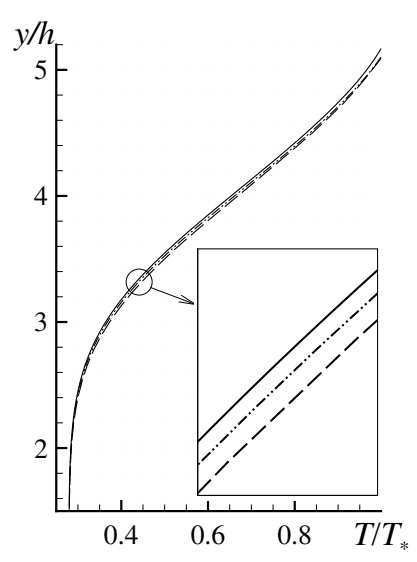

(b)

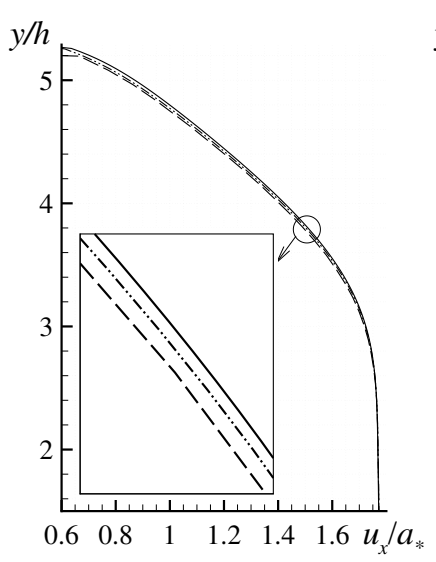

(c)

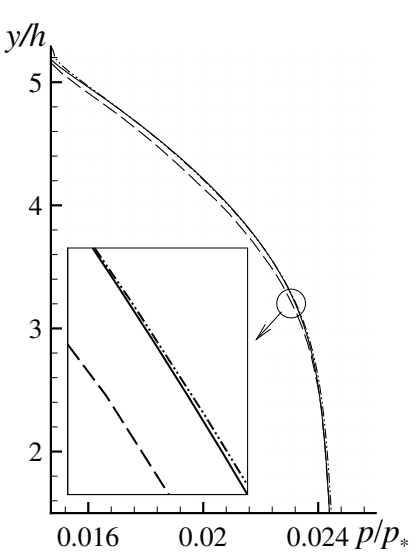

(d)

Figure 3: Plane nozzle. Distribution of gasdynamic parameters along the nozzle exit crosssection on different grids. 
Verification of the dispersed phase solver was conducted by testing the accuracy of integration of motion equations. The time step of the integration is chosen to ensure that it is smaller than the particle relaxation time and, simultaneously, the particle travel distance is smaller than the grid cell size. The estimate of the relaxation time $\tau$ is based on the Stokes formula for the drag and has the form

$$
\tau=\frac{2}{9} \frac{\rho_{p} r_{p}^{2}}{\mu}
$$

The test indicated that decreasing the time step by factors of 2,5 , and 10 does not have any effect on the particles trajectories. Therefore, the accuracy of integration of motion equations is sufficient for the chosen reference time step.

The final test was conducted to make sure that resulting trajectories are also independent of the resolution of the carrier phase flowfield. Figure 4 illustrates the convergence of the solution on the series of computational grids. The computations were performed on two grids, consisting of $608 \times 48$ and $1179 \times 96$ cells and the results were compared with the solution obtained on a very fine grid, consisting of $2184 \times 180$ cells. The difference in particle position for two coarser grids did not exceed $0.45 \%$ and $0.2 \%$ of nozzle exit width, respectively. We consider the resolution $2184 \times 180$ cells to be sufficient for the purposes of the present study. Therefore, the results reported in the following section were obtained on the latter, most refined grid.

\section{Results and discussion}

\subsection{Plane nozzle}

The local Mach number and temperature flowfields from the numerical simulation of the carrier phase are shown in Fig. 5. A thick boundary layer in the supersonic part of the nozzle, which is a typical feature of the flow in low-Reynolds number nozzles, is clearly visible. At higher Reynolds numbers, an internal shock is generally observed in wedge-like and conical nozzles as 


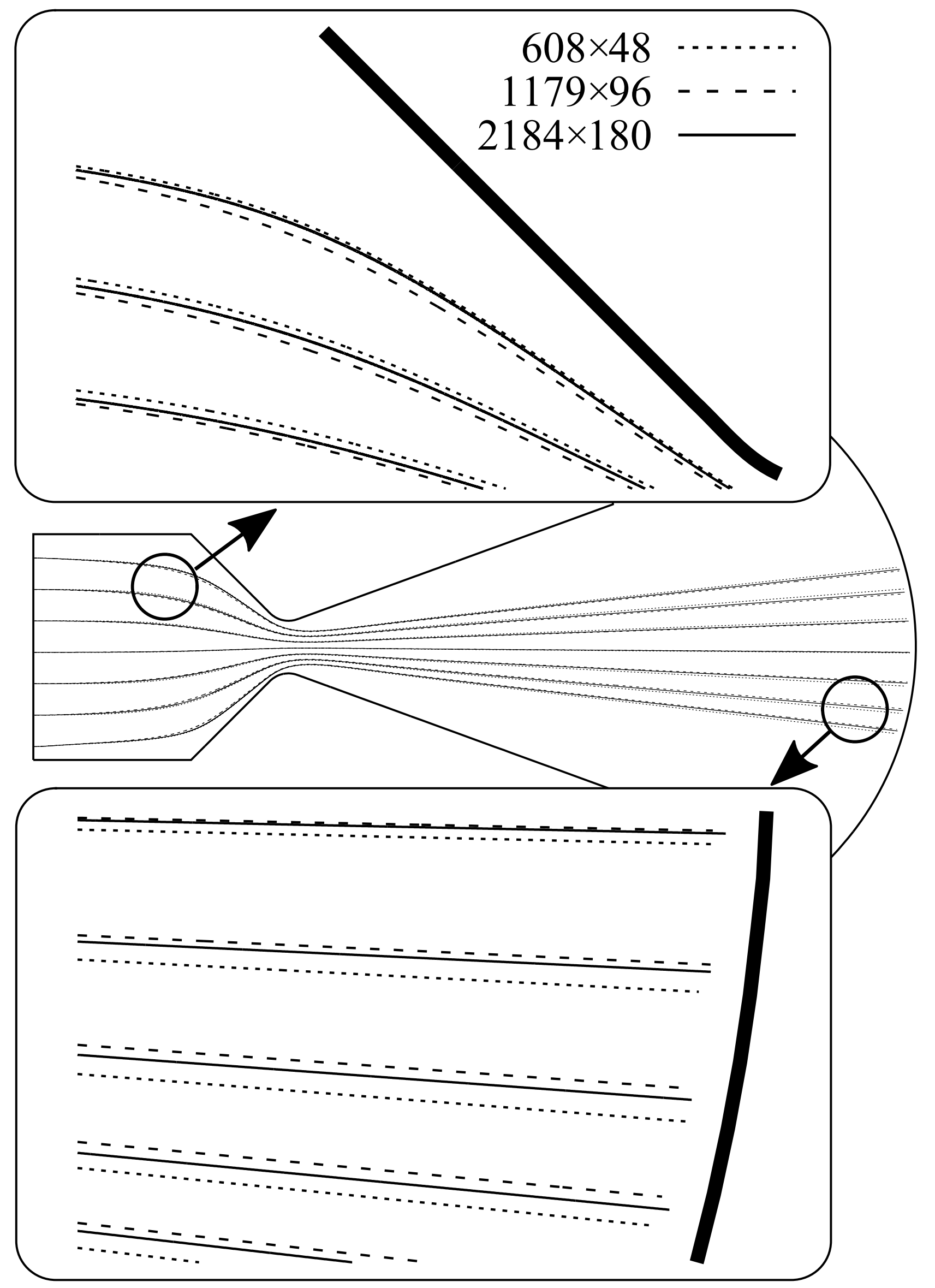

Figure 4: Trajectories of particles traced on flowfields with different spatial resolutions. 
a consequence of the discontinuity of the nozzle generatrix second derivative at the point where the wedge-shaped/conical part joins the nozzle rounding (Pirumov and Roslyakov, 1986). In the present case it is absent as a result of the smoothing effect of viscosity, which leads to a smooth profile of the boundary layer edge. The rapid growth of the boundary layer also reduces the effective nozzle cross-section and leads to a lower exit Mach number, $\mathrm{M}_{\text {exit }} \approx 3.33$ compared to the design value $\mathrm{M}_{d}=4$.

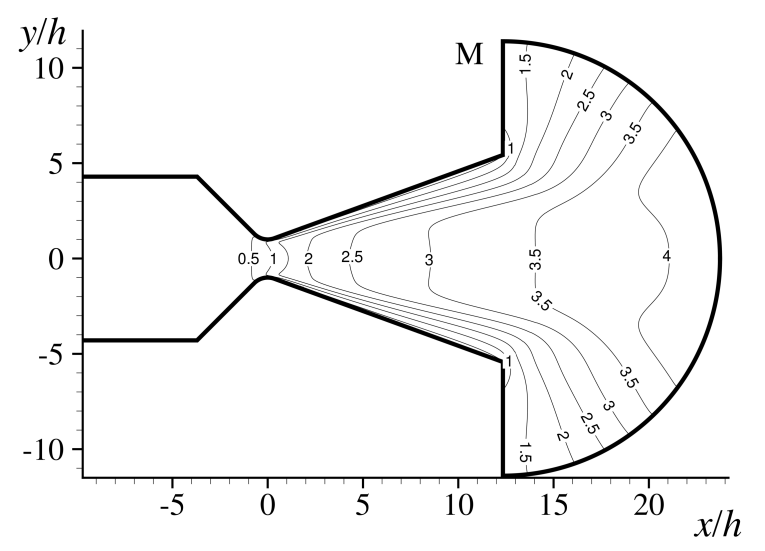

(a)

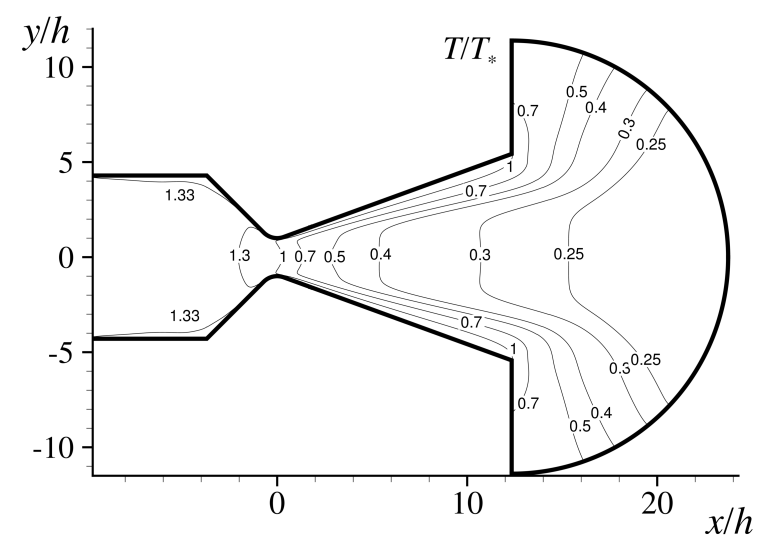

(b)

Figure 5: Plane nozzle. Isolines of local Mach number and temperature.

The computed trajectories of particles of different sizes are shown in Figs. 6a-6h. In each figure, the trajectories of 20 particles uniformly seeded in the prechamber are presented. As expected, the trajectories of large particles (such as $r_{p}=5 \mu \mathrm{m}$ ) are almost rectilinear, which means the particles are unaffected by the gas flow. For smaller particles the effect of aerodynamic focusing becomes pronounced. It is clearly visible that for the case $r_{p}=1.18 \mu \mathrm{m}$ particles in the central part of the beam are almost completely focused at the nozzle exit, while the peripheral part of the beam collided with the converging part of the nozzle (see Fig. 6c). On decreasing the particle radius, trajectory intersection appears on the symmetry line, behind which the whole beam expands (see Fig. 6d and 6e). With a decrease in the particle size, the point is gradually moving towards the nozzle throat. 


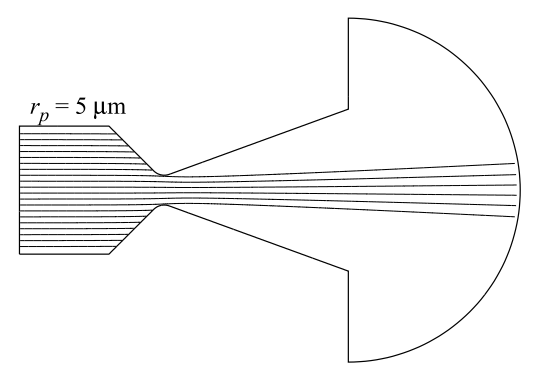

(a)

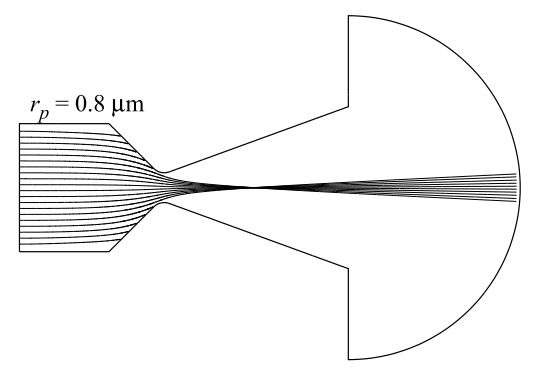

(d)

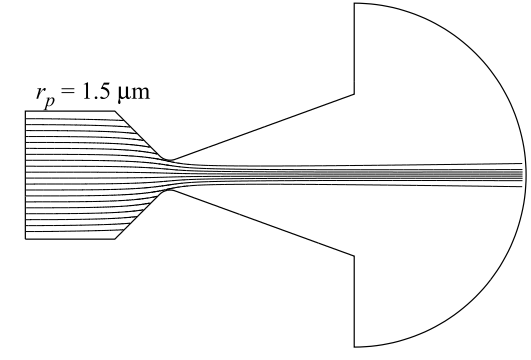

(b)

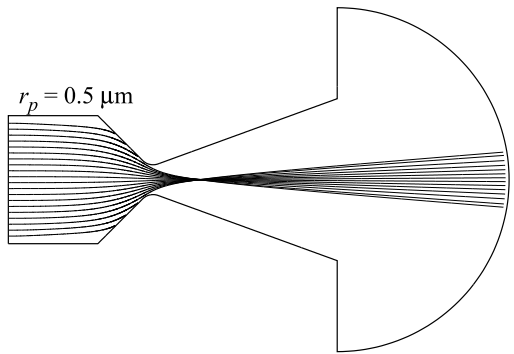

(e)

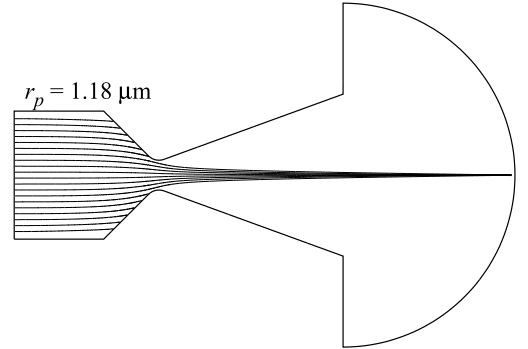

(c)

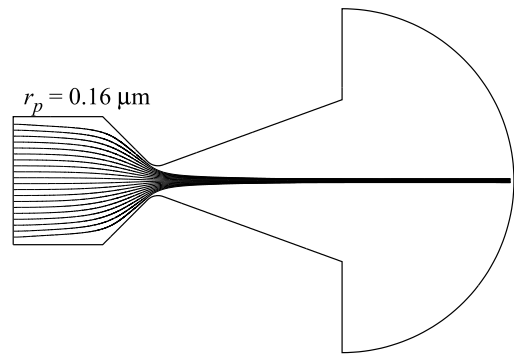

(f)

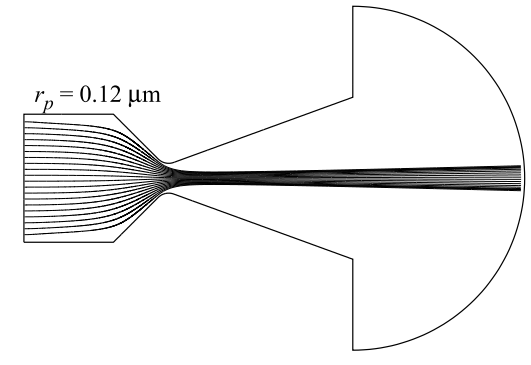

$(\mathrm{g})$

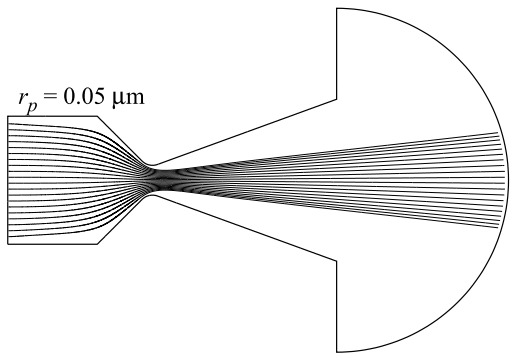

(h)

Figure 6: Plane nozzle. Trajectories of particles of different radii. 
Unexpected was the fact that a further decrease in the particle size leads to recurring aerodynamic focusing (Fig. 6f). In this case, the particle trajectories do not intersect the symmetry line and aerodynamic focusing affects not only particles seeded near the symmetry line but virtually all particles that entered the prechamber. For very small particles the effect of flow divergence in the supersonic part of the nozzle starts to have an impact and aerodynamic focusing does not occur.

These results demonstrate that aerodynamic focusing in the supersonic convergent-divergent nozzle is feasible and, moreover, it is observed for two different sizes of particles, $1.18 \mu \mathrm{m}$ and $0.12 \mu \mathrm{m}$ in the case considered.

One of the possible explanations for this result is that there are two different scenarios of of the collimation process. In the first case of relatively large and heavy particles, the influence of the carrier phase in the convergent part of the nozzle is sufficient to diverge particles at a small angle. The particles in the central part of the beam pass through the nozzle throat and focus at the nozzle exit, as is shown in Fig. 6c. The particles in the peripheral part of the beam hit the nozzle wall and, in our case, are excluded from the simulation. The diverged particles move in the supersonic part of the nozzle only due to inertial forces, because of the significant decrease in the carrier phase density and, consequently, the drag force acting on the particles. The smaller particles are diverged at greater angles, so the peripheral part of the initial beam also passes through the nozzle throat. However, these particles are heavy enough to be unaffected by the drag in the supersonic part of the nozzle. This results in a beam defocusing (see Figs. 6d-6e). For even smaller particles the drag in the diverging part of the nozzle (or at least in the region just downstream of the nozzle throat) become comparable to the inertial forces, and the forces in the converging and diverging part compensate each other, producing a focused beam.

Figure 7 shows the variation of the longitudinal velocity $u$ of both the carrier and dispersed phases as a function of the longitudinal coordinate $x$ for each of 20 particles whose trajectories 
are shown in Figs. 6c and 6f. Thus, each line corresponds to a particle with a specific initial distance from the nozzle axis. It is evident that in the case of large particles there is a strong velocity non-equilibrium between the two phases. The dispersed phase moves considerably slower than the gas in the supersonic part of the nozzle. It should be noted that the velocity of particles keeps growing in the divergent part, although at the nozzle exit it is 6 times lower than the gas velocity. Thus, it can be concluded that the use of supersonic nozzles allows one not only to generate a collimated beam of particles, but also to increase its velocity, compared to a convergent sonic nozzle. In terms of velocity, aerodynamic focusing is even more efficient for small particles. In this case the velocity non-equilibrium is much less pronounced, resulting in the formation of a collimated beam of particles with the velocity only $40 \%$ lower than the gas jet velocity. It should be noted, that in real-life applications you need to not only accelerate the particles up to a sufficiently high speed to make them stick to the surface but also to localize the area of effect, therefore, a highly collimated and high velocity beam is strongly desirable.

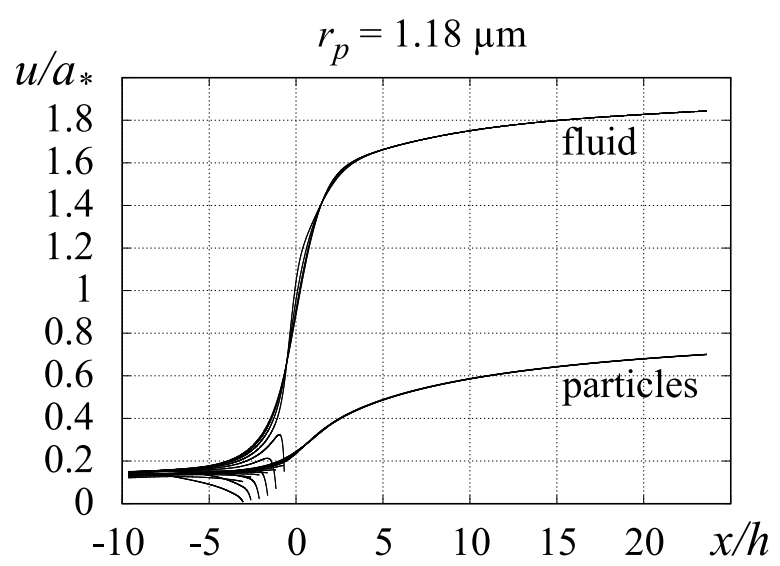

(a)

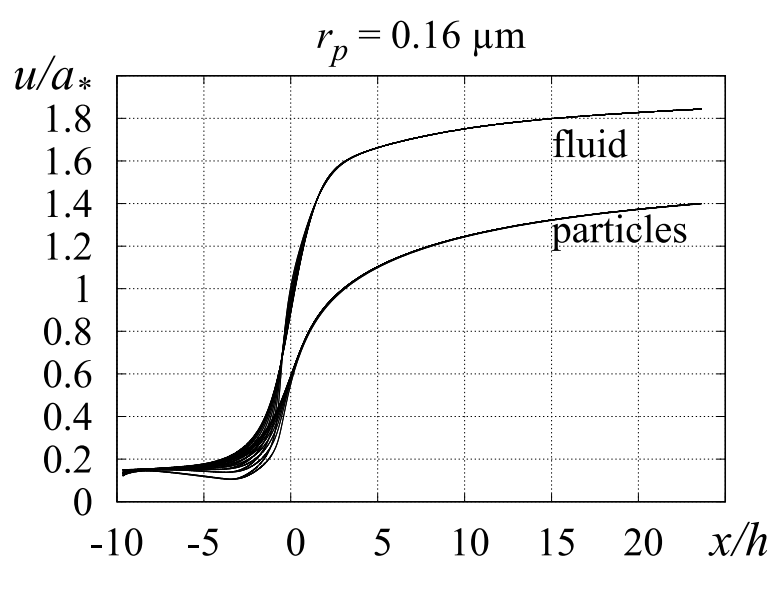

(b)

Figure 7: Plane nozzle. Distribution of longitudinal velocity of carrier and dispersed phases in cases of collimation.

Figure 8 shows the evolution of the particle Reynolds $\operatorname{Re}_{p}$ and the Mach $\mathrm{M}_{p}$ numbers along the trajectories. It is evident that the flow regimes around the particle change in a very wide 
range and, in the case of small particles, there is an extensive range corresponding to the free molecular flow. Thus, the equations for calculation of the drag force acting on the particle play a crucial role in numerical simulation of the problem under consideration and should beable to predict the drag correctly in a wide range of the regimes, from the creep flow to the free molecular flow.

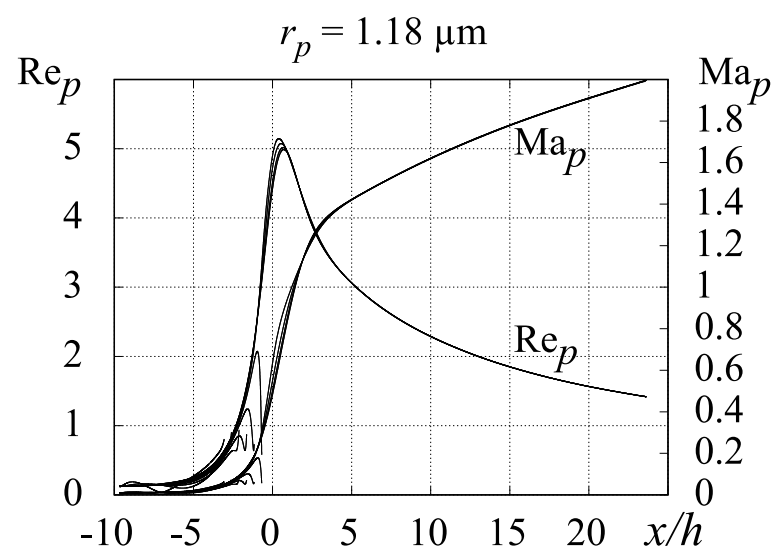

(a)

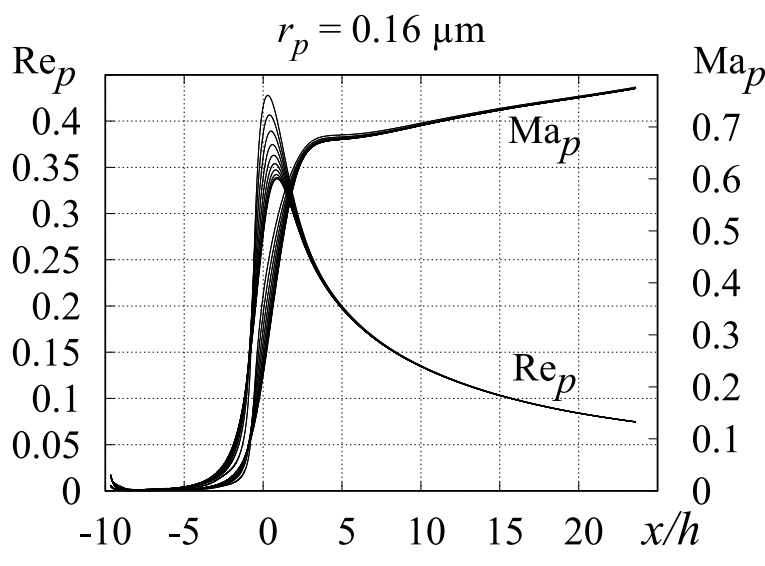

(b)

Figure 8: Plane nozzle. Evolution of the particle Mach number and particle Reynolds number along the trajectory in the cases of collimation.

\section{$3.2 \quad$ Axisymmetric nozzle}

The series of computations was repeated for the case of an axisymmetric nozzle with the same geometrical parameters, i.e. the nozzle had the same apex angle of the diverging part and same lengths of the prechamber, converging and diverging parts. Figure 9 shows the flowfields of the carrier gas. In terms of the boundary layer thickness and the absence of the internal shock, they look very similar to the flowfields in the wedge-shaped micronozzle. However, in the axisymmetric case the area of nozzle cross-section grows faster, resulting in higher both design Mach number $\mathrm{M}_{d} \approx 7.54$ of the nozzle and computed Mach number flow $\mathrm{M}_{\text {exit }} \approx 4.75$.

Numerical simulations shows that the patterns and relations obtained in the wedge-shaped 


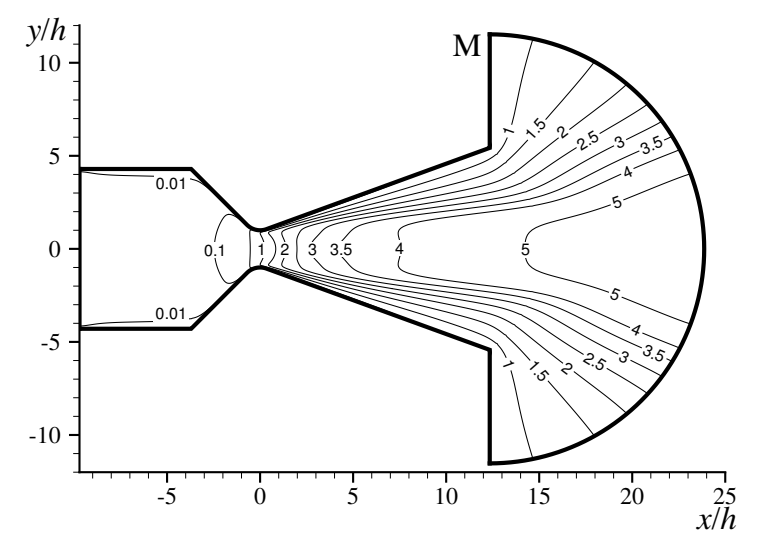

(a)

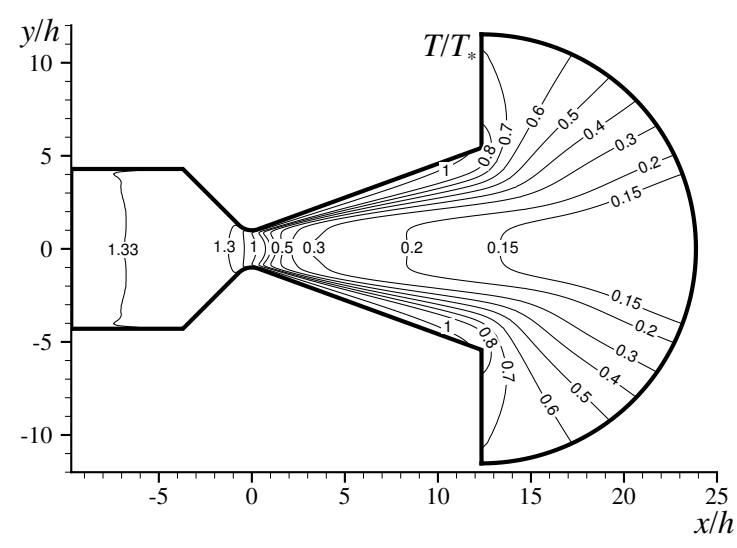

(b)

Figure 9: Axisymmetric nozzle. Isolines of the local Mach number and temperature.

micronozzle hold in the axisymmetrical case. There are two ranges of particle sizes for which aerodynamic focusing occurs. The collimation is observed for the $3.25 \mu \mathrm{m}$ and $0.2 \mu \mathrm{m}$ particles (see Fig. 10). Just like in the case of the plane micronozzle, there is also a strong velocity non-equilibrium between the phases, which decreases with a decrease in the particle size, as can be seen in Fig. 11.

Figure 12 illustrates the change in the flow regime around the particles along their trajectories. Note that the particle Mach number $\mathrm{M}_{p}$ is supersonic on an extensive segment of a trajectory for both size ranges.

Particle collimation in axisymmetric nozzles is arguably more important for practical applications because, unlike wedge-shaped nozzle, it allows them to be focused into a narrow beam rather than a plane sheet.

\subsection{D nozzle with a rectangular cross-section}

In practice, photolithographic etching produces three-dimensional wedge-shaped micronozzles with a finite width in the lateral direction and parallel lateral walls. So, in this Section the feasibility of particle focusing in a 3D nozle with a rectangular cross-section is studied. The 


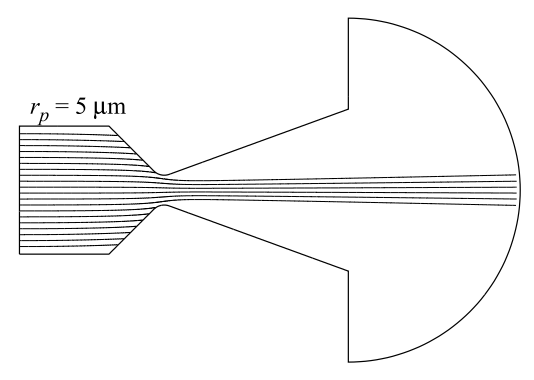

(a)

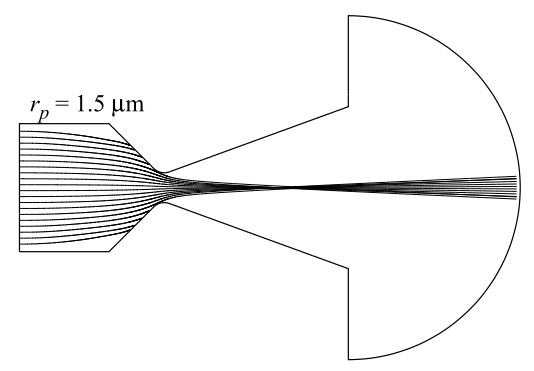

(d)

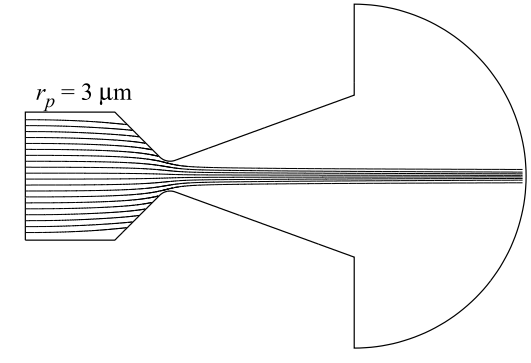

(b)

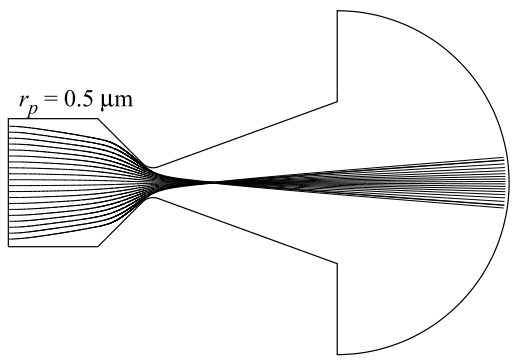

(e)

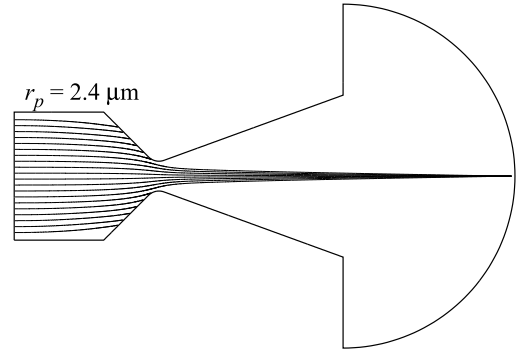

(c)

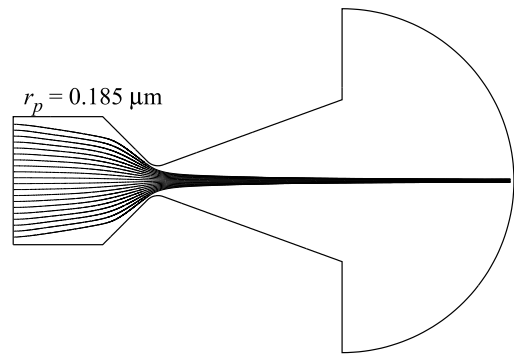

(f)

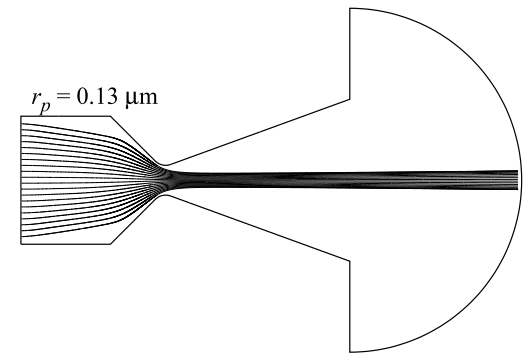

$(\mathrm{g})$

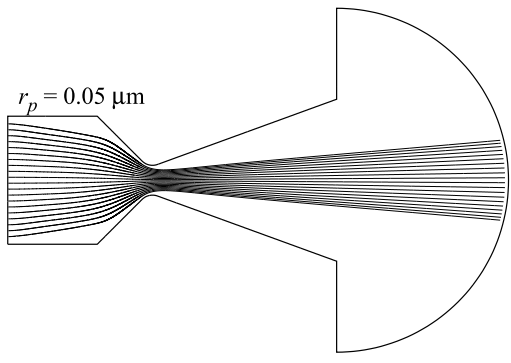

(h)

Figure 10: Axisymmteric nozzle. Trajectories of particles of different radii. 


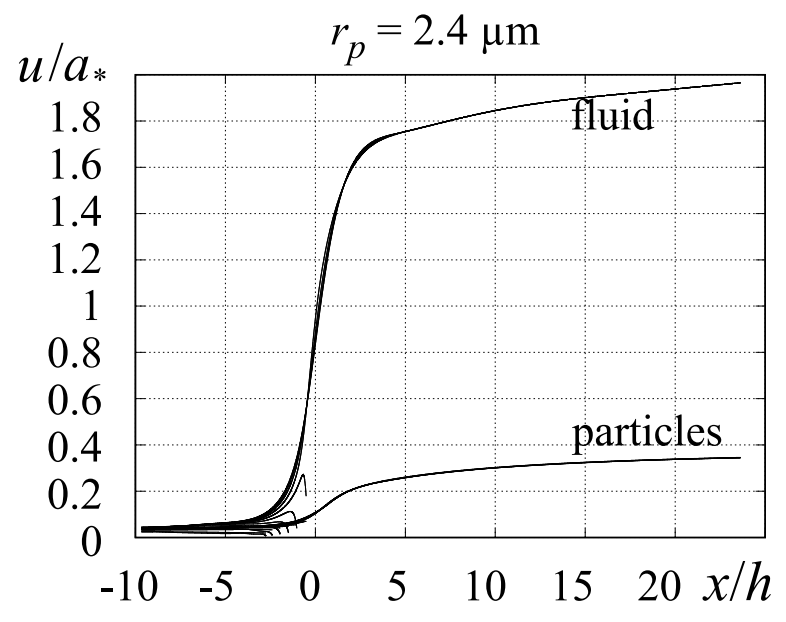

(a)

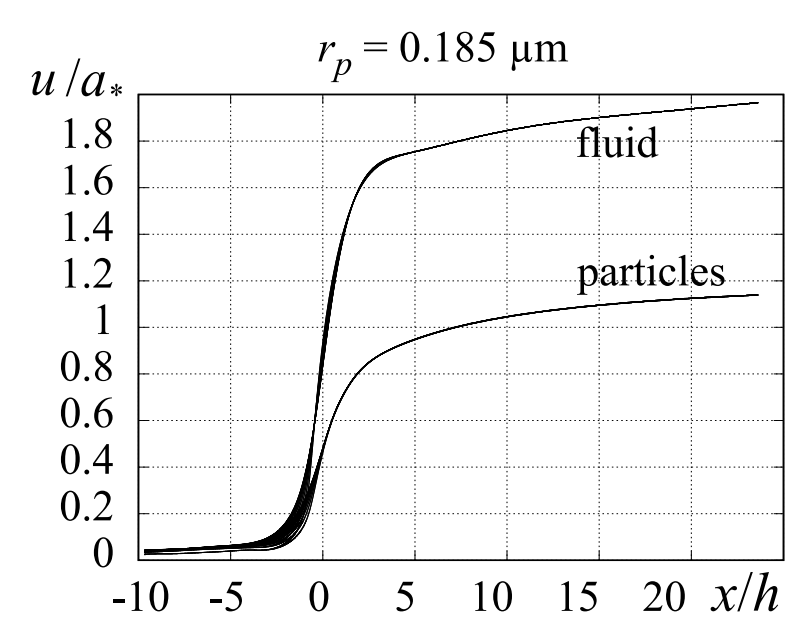

(b)

Figure 11: Axisymmteric nozzle. Distribution of the longitudinal velocity of the carrier and dispersed phases in the case of collimation.

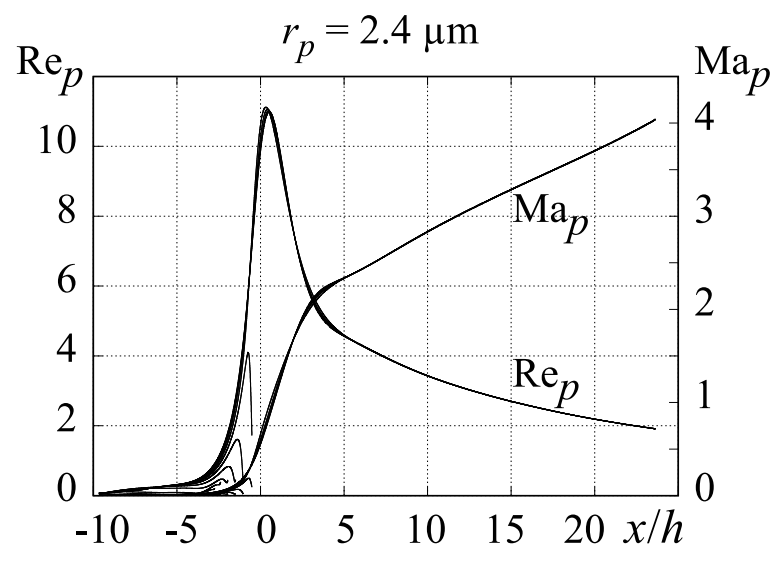

(a)

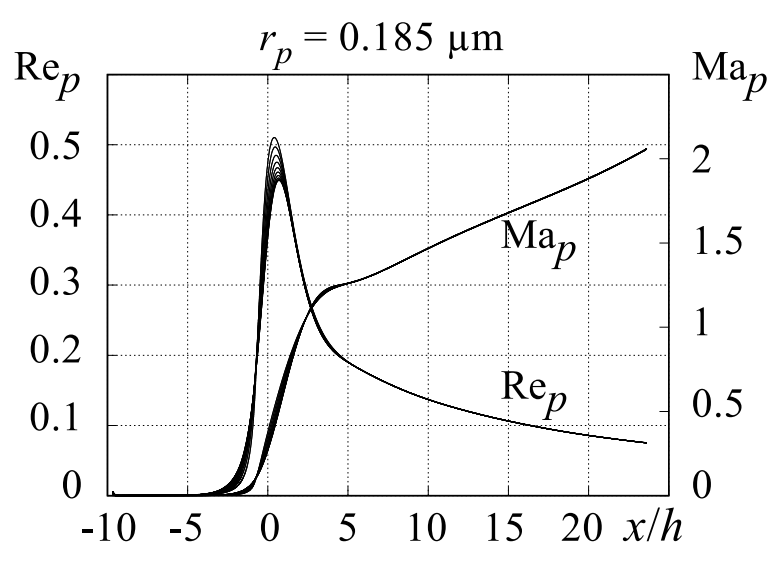

(b)

Figure 12: Axisymmetric nozzle. Evolution of the particle Mach number and particle Reynolds number along the trajectory in the case of collimation. 
main problem is focusing in the spanwise direction. The only factor that can contribute in beam focusing in the spanwise direction is the boundary layer growth on the lateral walls which results in displacement of flow streamlines from the wall.

Numerical simulations were performed for a nozzle with a relatively small ratio of the lateral width $D$ to to the nozzle exit transversal height $H, D / H=0.5$. It can be hoped that in this case the effects of aerodynamic focusing in the spanwise direction will be pronounced. The nozzle shape in the $X Y$ plane corresponded to that used in the $2 \mathrm{D}$ plane and axisymmetric cases. A computational grid consisting of $294 \times 40 \times 48$ cells was used in the numerical simulation of the carrier phase flowfield. Initial coordinates of particle trajectories form a rectangular array consisting of $21 \times 11$ equally spaced starting points.

Figure 13 shows a general view of a 3D wedge-shaped micronozzle with a rectangular crosssection and the local Mach number isolines. It can be seen that boundary layer growth on the lateral walls of the nozzle results in an even lower flow Mach number at the nozzle exit equal to $\mathrm{M}_{\text {exit }} \approx 2.86$. Figure 14 a shows behaviour of the particle trajectories in 3D nozzle. Additionally, the projections of the particle trajectories onto the planes $z=0$ and $y=0$ and are also shown in Figs 14b and 14c, respectively. It is evident that there is focusing of particles in the transversal direction. However, as can be seen in Fig. 14c, the effect of the boundary layer growth is rather weak. The particle trajectories are only partially diverted away from the parallel lateral walls in the supersonic part of the nozzle.

So it seems, that the main factor helping the focusing is convergence of nozzle walls (and, consequently, flow streamlines) in the subsonic part, and the boundary layer growth on the lateral walls is not sufficient to produce the desired effect of aerodynamic focusing. One may conclude that, for obtaining focusing in both transversal and lateral directions, the subsonic part of the nozzle also should be convergent in both these directions, similar to the axisymmetric nozzle. On the other hand, the shape of 3D nozzle with subsonic part converging in 
both transversal and spanwise directions is very unusual, especially if we consider micronozzles fabricated using photolithography/etching.

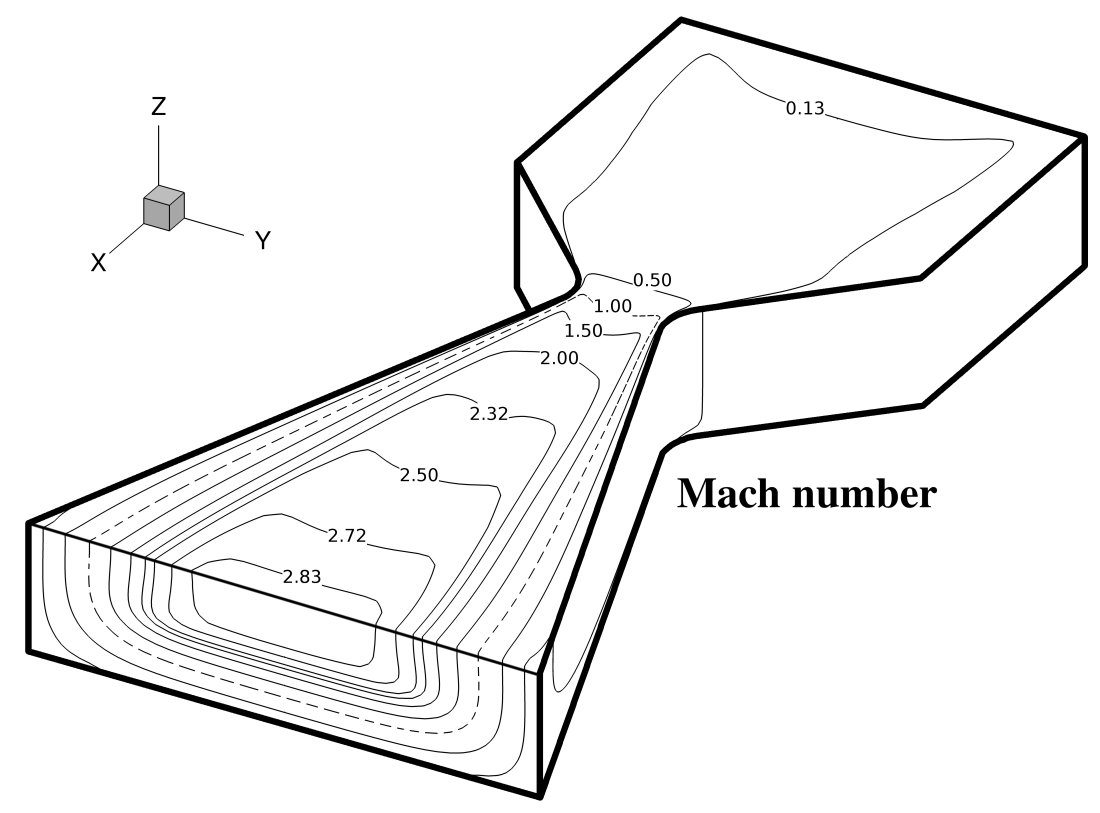

Figure 13: 3D nozzle. Isolines of the local Mach number.

\section{Conclusion}

Numerical simulations of particulate flows in supersonic (convergent/divergent) plane, axisymmetric, and 3D wedge-like nozzle with a rectangular cross-section were performed. It was shown that aerodynamic focusing of particles into a collimated beam can be obtained. An unexpected finding was that aerodynamic focusing is observed for two different sizes of particles — for relatively large particles $(1-3 \mu \mathrm{m})$ and for much smaller ones $(0.1-0.2 \mu \mathrm{m})$. In the former case, only particles in the core flow are focused, while for smaller particles the collimated beam includes nearly all particles entering the nozzle prechamber.

In both cases the particles continue to accelerate in the divergent part of the nozzle, though for smaller particles this process is much more efficient so that they exit the nozzle with the velocity only $40 \%$ lower than the fluid velocity. Thus, the use of supersonic micronozzles allows 


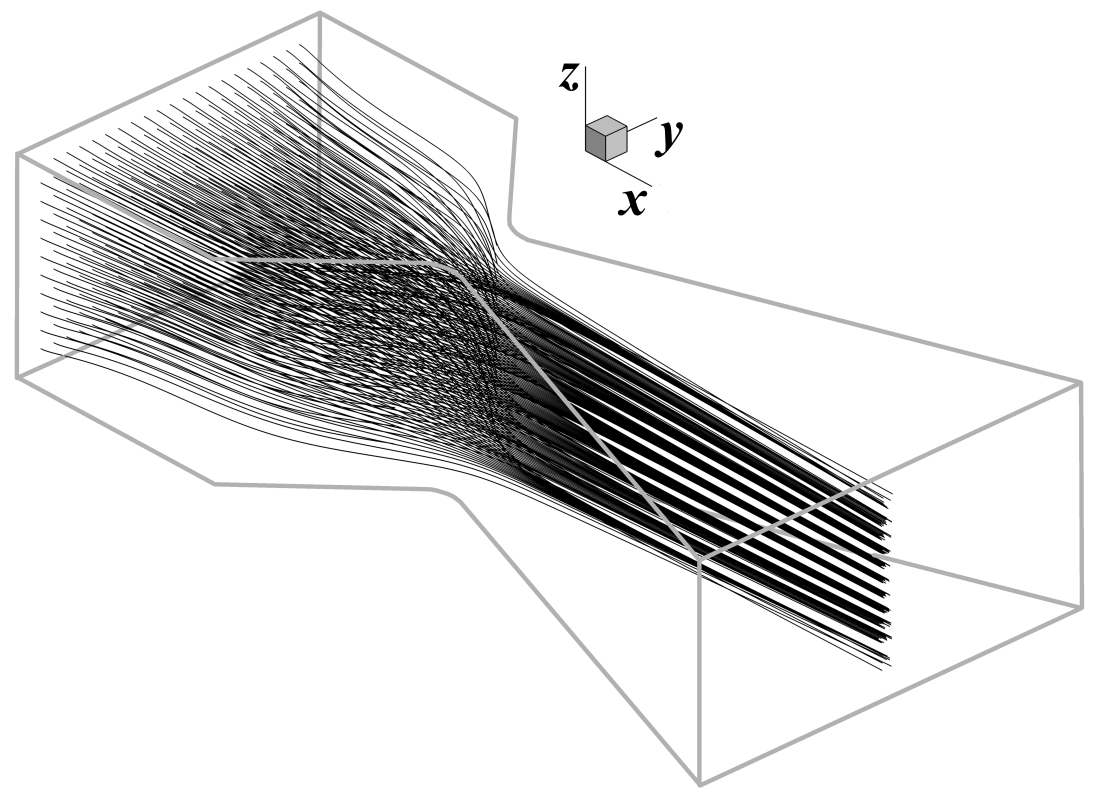

(a)

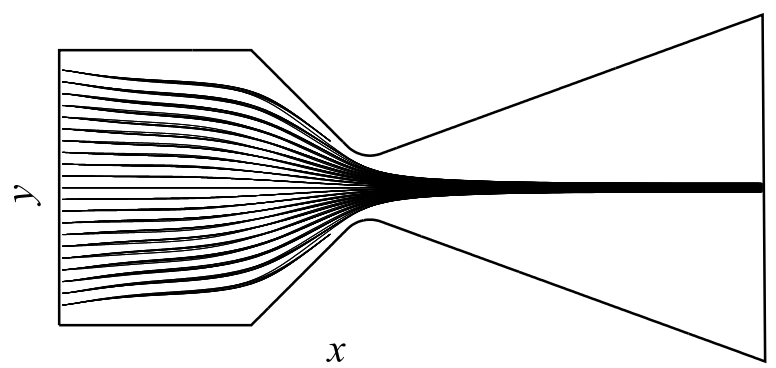

(b)

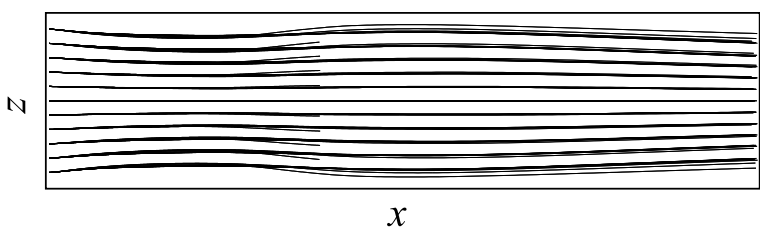

(c)

Figure 14: 3D nozzle. Trajectories of $0.135 \mu \mathrm{m}$ particles. 
one not only to generate a collimated particle beam but also to increase its velocity substantially in comparison to subsonic nozzles.

In a 3D nozzle with a rectangular cross-section focusing is only feasible in the transversal direction. The displacement of streamlines in the supersonic part of the nozzle caused by the growth of the boundary layer thickness on the lateral walls is not sufficient for beam collimation.

Acknowledgements The work of Alexey Kudryavrsev and Anton Shershnev was supported by the Russian Science Foundation (grant No. 18-11-00246). This support is gratefully acknowledged.

Oyuna Rybdylova is grateful to the EPSRC, UK (grant EP/R012024/1) and Royal Society (UK) (grant IE 160014) for their financial support.

The authors would also like to thank Prof. A.N. Osiptsov for the fruitful discussion of the presented results.

\section{References}

Akhatov IS, Hoey JM, Thompson D, Lufturakhmanov A, Mahmud Z, Swenson OF, Schulz DL, Osiptsov AN (2009) Aerosol flow through a micro-capillary. Proc. of the ASME 2nd Micro/Nanoscale Heat \& Mass Transfer International Conference (MNHMT2009, December 18-21, 2009, Shanghai, China). Paper MNHMT2009-18421

Aniskin VM, Maslov AA, Mironov SG (2011) Effect of nozzle size on supersonic microjet length. Tech Phys Lett 37(11):1046-8. doi:10.1134/S1063785011110198

Bhattacharya S, Lufturakhmanov A, Hoey JM, Swenson OF, Mahmud Z, Akhatov IS (2013) Aerosol flow through a converging-diverging micro-nozzle. Nonlinear Engineering 2:103-12. 
Bird GA (1994) Molecular gas dynamics and direct simulation of gas flows. Clarendon Press, Oxford.

Boothroyd RG (1971) Flowing gas-solids suspensions. Ghapman and Hall Ltd., London.

Crowe CT, Babcock W, Willoughby PG (1973) Drag coefficient for particles in rarefied, low Mach numbers flows. Prog Heat and Mass Transf 6:419-28. doi:10.1016/B978-0-08-017035$0.50027-6$

Dandy DS and Dwyer HA (1990) A sphere in a shear flow at finite Reynolds number: effect of shear on particle lift, drag and heat transfer. J Fluid Mech 216: 381-410.

Gad-el-Hak M (1999) The fluid mechanics of microdevices — the Freeman scholar lecture. J Fluids Eng 21:5-33. doi:10.1115/1.2822013

Henderson CB (1976) Drag coefficients for spheres in continuum and rarefied flows. AIAA J 14:707-8. doi:10.2514/3.61409

Ho C-M, Tai Y-C (1998) Micro-electro-mechanical systems (MEMS) and fluid flows. Annu Rev Fluid Mech 30:579-612. doi:10.1146/annurev.fluid.30.1.579

Ivanov MS, Markelov GN, Ketsdever AD, Wadsworth DC (1999) Numerical study of cold gas micronozzle flows. AIAA Paper No. 99-0166, 15 p. doi:10.2514/6.1999-166

Karniadakis G, Beskok A, Aluru N (2005) Microflows and nanoflows. Springer, N.Y.

Ketsdever A, Wadsworth DC, Wapner PhG, Ivanov MS, Markelov GN (1999) Fabrication and predicted performance of conical DeLavale micronozzles. AIAA Paper No. 99-2724. 12 p. doi:10.2514/6.1999-2724

Kiselev SP, Kiselev VP (2009) Numerical simulation of nanoparticle acceleration in a Laval 
micronozzle with subsequent deceleration in a wall compression layer. Int J Aerospace Innovations. 1(3):117-27.

Kogan MN (1969) Rarefied Gas Dynamics. Plenum, New York.

Ling Y, Haselbacher A, Balachandar S (2011) Importance of unsteady contributions to force and heating for particles in compressible flows: Part 1: Modeling and analysis for shock-particle interaction Int J Multiph Flow 37(9):1026-44 doi:10.1016/j.ijmultiphaseflow.2011.07.001

Louwerse MC (2009) Cold gas micro propulsion. University of Twente, $176 \mathrm{p}$.

Lutfurakhmanov A, Loken G, Schulz DL, Akhatov IS (2010) Capillary-based liquid microdroplet deposition. Appl Phys Lett 97:124107. doi:10.1063/1.3489918

McLaughlin JB (1991) Inertial migration of a small sphere in linear shear flow. J Fluid Mech 224: $261-74$.

Molleson GV, Stasenko AL (2008) Acceleration of microparticles in a gasdynamic facility with high expansion of flow. High Temperature 46(1):100-7.

de la Mora JF, Riesco-Chueca P (1988) Aerodynamic focusing of particles in a carrier gas. J Fluid Mech 195:1-21. doi:10.1017/S0022112088002307

Nigmatulin RI (1990) Dynamics of Multiphase Media. Taylor\&Francis, Washington.

Papyrin A, Kosarev V, Klinkov S, Alkhimov A, Fomin V (2007) Cold Spray Technologies. Elsevier, Amsterdam.

Phalnikar KA, Kumar R, Alvi FS (2008) Experiments on free and impinging supersonic microjets. Exp Fluids 44(5):819-30 doi:10.1007/s00348-007-0438-4

Pirumov UG, Roslyakov GS (1986) Gas Flow in Nozzles. Springer-Verlag, Berlin, Heidelberg, New York, Tokyo. 
Prosperetti A (2007) Averaged equations for multiphase flow. In: Prosperetti A (ed), Tryggvason G (ed) Computational Methods for Multiphase Flow. Cambridge University Press, New York, pp 237-281.

Rothe DE (1971) Electron-beam studies of viscous flow in supersonic nozzles. AIAA J 9(5):80411. doi:10.2514/3.6279

Rybdylova O, Lebedeva N, Kudryavtsev A, Shershnev A (2013) Aerodynamic focusing of inertial particles in supersonic micronozzles. Proc Appl Math Mech 13:503-4. doi:10.1002/pamm.201310244

Saffman PG (1965) The lift on a small sphere in a slow shear flow. J Fluid Mech 22, part $2: 385-400$

Sebastião IB, Santos WFN (2014) Numerical simulation of heat transfer and pressure distributions in micronozzles with surface discontinuities on the divergent contour. Computers \& Fluids 92:125-37. doi:10.1016/j.compfluid.2013.12.023

Shershnev AA, Kudryavtsev AN (2015) Kinetic simulation of near field of plume exhausting from a plane micronozzle. Microfluid Nanofluid 19(1):105-15. doi:10.1007/s10404-015-1553-9

Squires K (2007) Point-particle methods for disperse flows. In: Prosperetti A (ed), Tryggvason G (ed) Computational Methods for Multiphase Flow. Cambridge University Press, New York, pp 282-319.

Stasenko AL, Chirikhin AV (2002) The evaporation of quartz particles behind strong shock waves in slightly dusty air. High Temperature 40(6):865-71.

Sternin LE (1974) Principles of Gas Dynamics of Two-Phase Flows in Nozzles (in Russian). Mashinostroenie, Moscow. 
Sutherland GS, Maes ME (1966) A review of microrocket technology: $10^{-6}$ to 1 lbf thrust. J Spacecraft and Rockets 3(8):1153-65. doi:10.2514/3.28621 